\title{
Estrategias participativas y las prácticas sociales de la comunidad nativa de Tsuntsuntsa, Amazonas, Perú, 2019
}

\section{Participatory strategies and social practices of the native community of Tsuntsuntsa, Amazonas, Peru, 2019}

\author{
Cecil Wilmer Burga Campos
}

\section{RESUMEN}

conservar las prácticas sociales de la Comunidad Nativa Tsuntsuntsa, Bagua, Amazonas, Perú, 2019. La investigación tuvo un diseño no experimental con un solo grupo, se trabajó con una muestra conformada por 24 pobladores equivalente al 10\% de la población total de la comunidad. Se aplicó una encuesta para la evaluación de las dimensiones de la variable estrategias participativas: actividades preparatorias, exposición de conocimientos y participación, y las dimensiones de la variable prácticas sociales: saberes prácticos, habilidades culturales y materialidades, en los niveles mala, regular, bueno, excelente. Los resultados evidencian la valoración de nivel mala de las dimensiones, actividades preparatorias en un $62.5 \%$, exposición de conocimientos en un $66.7 \%$ y participación en un $58.3 \%$, saberes prácticos en un $66.7 \%$, habilidades culturales el 100\%, la valoración de nivel regular de las dimensiones, actividades preparatorias en un 37.5\%, exposición de conocimientos en un 33.3\%, participación en un $41.7 \%$, saberes prácticos en un 33.3\%, la valoración de nivel bueno solo en la dimensión materialidades con el $79.2 \%$ y el $20.8 \%$ en el nivel excelente. Se concluye que existe un bajo nivel en todas las dimensiones de las estrategias participativas y las prácticas sociales de la Comunidad Nativa Tsuntsuntsa, considerando que la comunidad tiene un gran desafío en todas sus dimensiones para conservar sus prácticas sociales. (Hasta 200 palabras)

Palabras clave: Estrategias participativas, actividades preparatorias.

\begin{abstract}
This research aimed to identify participatory strategies that contribute to the conservation of social practices in the Tsuntsuntsa Native Community, Bagua, Amazon, Peru, 2019. The research had a non-experimental design with only one group, we worked with a sample conformed by 24 inhabitants equivalent to $10 \%$ of the total population of the community. A survey was applied to evaluate the dimensions of the variable participatory strategies: preparatory activities, exposure of knowledge and participation, and the dimensions of the variable social practices: practical knowledge, cultural skills and materialities, at the levels of bad, regular, good, excellent. The results show a poor level of assessment of the dimensions, preparatory activities in $62.5 \%$, exposure of knowledge in $66.7 \%$ and participation in $58.3 \%$, practical knowledge in $66.7 \%$, cultural skills in $100 \%$, the assessment of regular level of the dimensions, preparatory activities in $37.5 \%$, exposure of knowledge in $33.3 \%$, participation in $41.7 \%$, practical knowledge in $33.3 \%$, the assessment of good level only in the dimension materialities with $79.2 \%$ and $20.8 \%$ in the excellent level. It is concluded that there is a low level in all dimensions of participatory strategies and social practices of the Tsuntsuntsa Native Community, considering that the community has a great challenge in all its dimensions to preserve its social practices.
\end{abstract}

Keywords: Participatory strategies, preparatory activities.

1 Docente auxiliar de la Facultad de Ciencias Económicas y Administrativas de la Universidad Nacional Toribio Rodríguez de Mendoza de Amazonas. Correo electrónico: cewilburgac@yahoo.es 


\section{I.INTRODUCCIÓN}

En las últimas décadas, numerosas investigaciones nacionales e internacionales vinculadas a las estrategias participativas y las prácticas sociales, han mostrado su preocupación por las crisis sociales de las comunidades nativas, ocasionado por la pérdida de los valores culturales de los grupos étnicos y su integración a ella, utilizando estrategias participativas desarrollados por instituciones nacionales; para tratar de minimizar y revertir esta crisis y a su vez apoyar a las comunidades locales a desarrollarse sosteniblemente.

Asimismo teniendo en cuenta que la realidad cultural en el mundo entero viene siendo afectada como consecuencia de la globalización, debido a la falta de normas que permitan la conservación cultural originaria de los pobladores que de generación en generación han venido poniendo en práctica un legado importante de costumbres, tradiciones, lengua, que hacen singular a cada pueblo originario, al poner de manifiesto su cultura a través de las diferentes prácticas sociales que los identifica como tales.

Cuando nos referimos a prácticas sociales definitivamente nos referimos a toda acción que los pobladores de una determinada comunidad ponen en práctica a fin de diferenciarse de los demás, acontecimientos que se ponen de manifiesto a través del habla, danza, religión, costumbres, tradiciones, curanderismo, manejo y uso de plantas medicinales, vivienda, artesanía canto, entre otras prácticas puestas de manifiesto en reuniones familiares $\mathrm{o}$ comunales con puesta en común de estrategias participativas pertinentes.

De la misma manera lo afirma De Gortari (2000), las prácticas sociales se refieren a un conjunto de trabajos, acciones, ejercicios, que se realizan constantemente dentro de una sociedad.

Según Carmen (2013), las estrategias participativas constituyen un conjunto de acciones integradoras e integradas entre sí, basadas en interacción y realizadas por los aprendices con la orientación del instructor. Asimismo la universidad como ente rector de la investigación científica, promueve la investigación con la finalidad de contribuir con el cambio de la sociedad, es por ello que considerando la existencia pluricultural de la región Amazonas, y observando la existencia de comunidades nativas de origen awajún que se viene debilitando debido al ingreso de la población hispana en el territorio awajún, y, con la finalidad de contribuir en preservar las prácticas sociales de la comunidad nativa de Tsuntsuntsa-Aramango-Bagua, a través de la identificación de estrategias participativas que deben ser puestas en práctica por la población. En ese sentido con el fin de promover las prácticas sociales de la comunidad de nativa de Tsuntsuntsa, se identificarán estrategias participativas para preservar las prácticas sociales de la Comunidad Nativa
Tsuntsuntsa-Aramango-Bagua, 2019, investigación que debe conllevar a rescatar vivencias y costumbres ancestrales practicadas por los pobladores awajún. En tal sentido considerando que la comunidad Nativa de Tsuntsuntsa es muy rica y basta en su cultura originaria pero lamentablemente, lentamente ha venido siendo afectada por la colonización de pobladores hispanos en el entorno comunal, han dado lugar para que sus prácticas sociales originarias vayan siendo desplazadas por otras externas. Por eso al encontrar esta realidad problemática se desarrolla la investigación titulada: Estrategias participativas y las prácticas sociales de la comunidad nativa de Tsuntsuntsa-Aramango-Bagua-Amazonas, 2019; la cual precisa como problema de investigación: ¿Cuáles son las estrategias participativas que contribuyen a conservar las prácticas sociales de la Comunidad Nativa Tsuntsuntsa-Aramango-Bagua, 2019?, investigación que se orientó a identificar las estrategias participativas y las prácticas sociales de la Comunidad nativa de Tsuntsuntsa para conservar y preservar su sostenibilidad.

\section{MATERIAL Y MÉTODOS}

La investigación fue no experimental de tipo descriptiva simple. En la variable independiente estrategias participativas, se tiene 3 dimensiones y 12 preguntas, en la variable dependiente prácticas sociales, se tiene 3 dimensiones y 12 preguntas, que a continuación se pasa a detallar para cumplir con los objetivos propuestos en la presente investigación. Se utilizó el siguiente diseño de investigación (Hernández, Fernández, y Baptista, 2006)

\section{Donde}

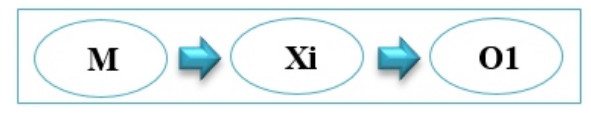

\section{M: Muestra}

Xi: Estrategias participativas que contribuyan a conservar las prácticas sociales.

O1: Resultado de la medición.

Población: Estuvo conformada por 240 pobladores de la comunidad de Tsuntsuntsa del distrito Aramango, provincia de Bagua, departamento Amazonas.

Muestra: La muestra estuvo conformada por el 10\% de la población total, correspondiente a 24 pobladores. Se utilizó un muestreo no probabilístico de tipo intencional.

Métodos:

Inductivo: Este proceso implicó obtener información particular de un grupo de individuos de la comunidad nativa de Tsuntsuntsa (Sierra, 2012). 
Etnográfico: A través de este proceso se buscó conocer la vida cotidiana del grupo muestra desde el interior del mismo, identificando y describiendo sus rasgos cualitativos (Murillo y Martínez, 2010).

Procedimiento: El desarrollo de la investigación tuvo como base la identificación de las estrategias participativas que contribuyan a conservar las prácticas sociales de la Comunidad Nativa Tsuntsuntsa-Aramango-Bagua, 2019, la misma que se inició con la observación, luego se realizó el diseño y análisis de la investigación, considerando los fundamentos teóricos que sirvieron de base para la realización de la investigación.

a.Primera fase: Validación y confiabilidad del instrumento.

b.Validación del instrumento: La encuesta fue validada por tres expertos con amplio conocimiento en el tema.

Confiabilidad del instrumento: La confiabilidad del instrumento se realizó mediante el grupo de estudio de la comunidad teniendo las mismas características de la muestra, etnia, lengua, costumbres, tradiciones entre otros; datos que fueron procesados mediante el software estadístico SPSS, a través del coeficiente alfa de Cronbach dando un alto grado de confiabilidad en todas sus dimensiones.

\section{ESTRATEGIAS PARTICIPATIVAS}

\begin{tabular}{|c|c|}
\hline \multicolumn{2}{|c|}{ Estadísticos de fiabilidad } \\
\hline $\begin{array}{c}\text { Alfa de } \\
\text { Cronbach }\end{array}$ & $\mathrm{N}^{\circ}$ de elementos \\
\hline, 815 & 12 \\
\hline
\end{tabular}

PRÁCTICAS SOCIALES

\begin{tabular}{|c|c|}
\hline \multicolumn{2}{|c|}{ Estadísticos de fiabilidad } \\
\hline $\begin{array}{c}\text { Alfa de } \\
\text { Cronbach }\end{array}$ & $\mathrm{N}^{\circ}$ de elementos \\
\hline, 868 & 12 \\
\hline
\end{tabular}

c. Segunda fase: Análisis descriptiva simple: Los datos que se recogieron de la encuesta, fueron procesados y analizados utilizando la estadística descriptiva simple.

d. Tercera fase: Estadística descriptiva inferencial: Se recogieron los datos de la estadística descriptiva simple y fueron procesados a través de los programas estadísticos de SPSS y EXCEL para su respectiva interpretación en tablas y figuras de acuerdo a los resultados alcanzado por dimensiones.

\section{RESULTADOS}

\section{DIMENSIÓN 1: ACTIVIDADES PREPARATORIAS}

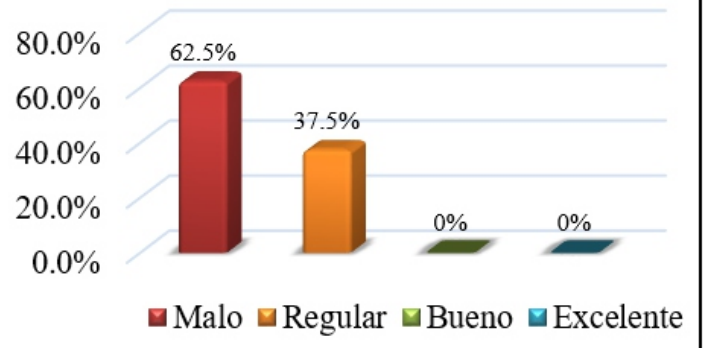

Figura 1. Porcentaje de los niveles de las actividades preparatorias.

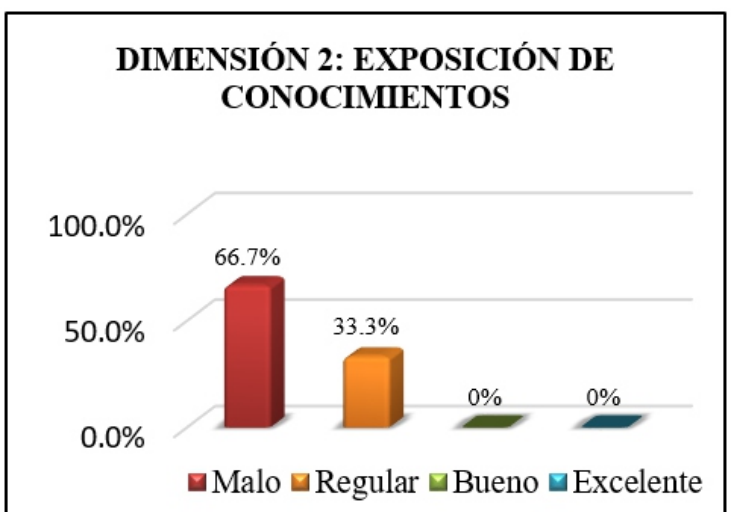

Figura 2. Porcentaje de los niveles de exposición de conocimientos.

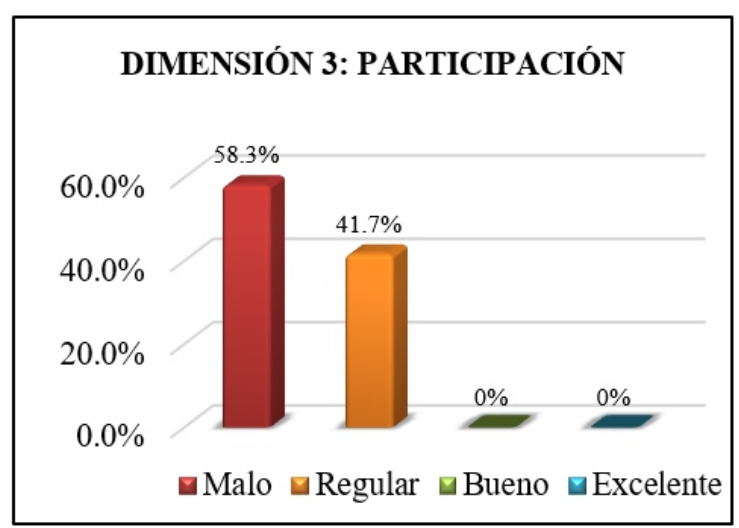

Figura 3. Porcentaje de los niveles de participación. 


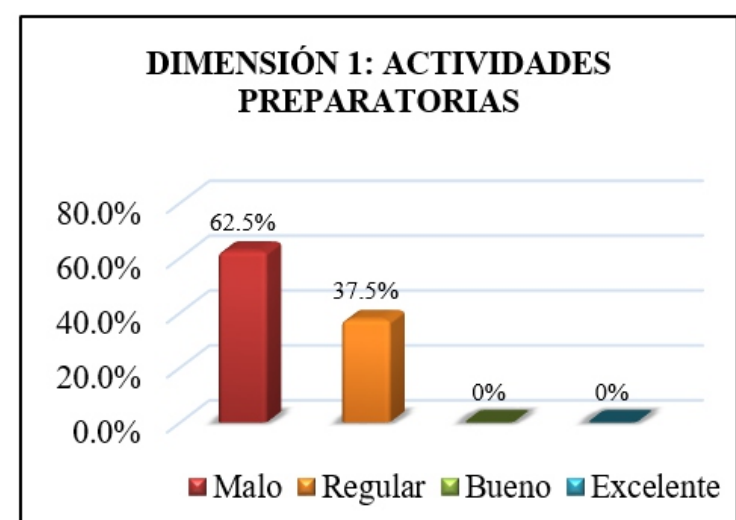

Figura 4. Porcentaje de los niveles de los saberes prácticos.

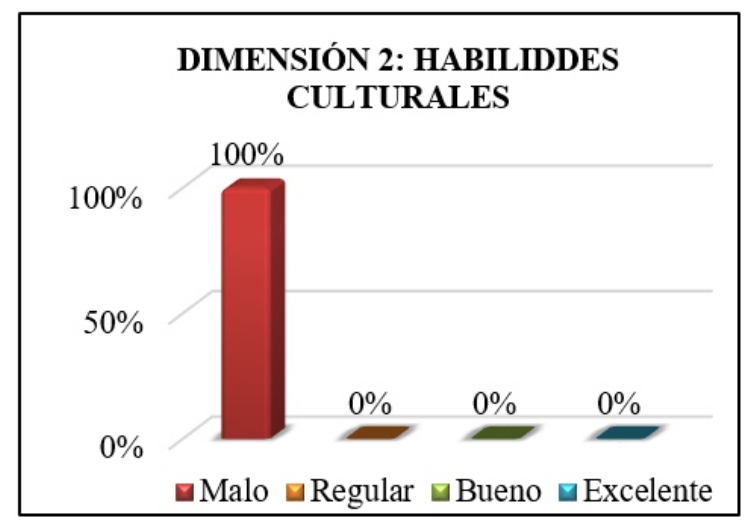

Figura 5. Porcentaje de los niveles de habilidades culturales.

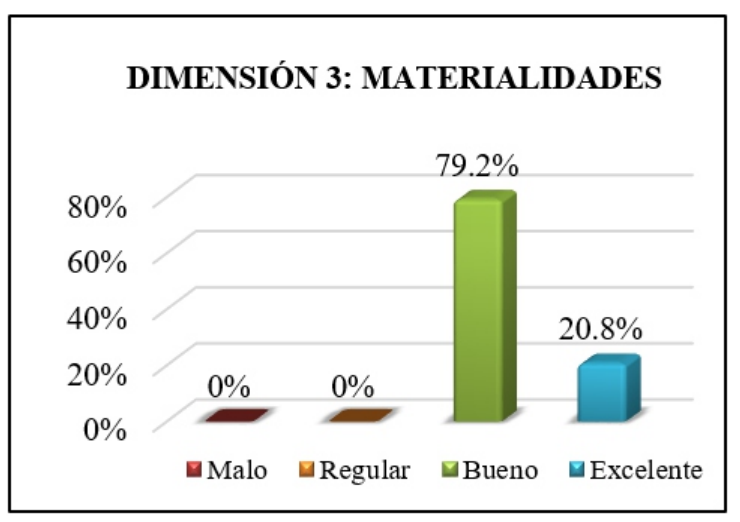

Figura 6. Porcentaje de los niveles de materialidades.

\section{DISCUSIÓN}

Las dimensiones o componentes de las prácticas sociales son importantes, ya que estos permiten conservar las mismas.

En la presente investigación en relación a la dimensión actividades preparatorias se identificó que de las 24 personas encuestadas de la comunidad nativa de Tsuntsuntsa el $62.5 \%$ que representa a 15 pobladores manifiestan que en la comunidad nativa hay un nivel malo respecto a actividades preparatorias y que el $37.5 \%$ que representan a 9 pobladores manifiestan que el nivel de actividades preparatorias es regular (Figura 1). Con respecto a la dimensión exposición de conocimientos se identificó que de las 24 personas encuestadas de la comunidad nativa de Tsuntsuntsa el $66.7 \%$ que representa a 16 pobladores quienes manifiestan que el nivel de conocimiento está en un nivel malo y que el 33.3\% que equivale a 8 pobladores manifiestan que el nivel de exposición de conocimientos de la comunidad alcanzando un nivel regular (Figura 2). Y con respecto a la dimensión participación se determinó que de las 24 personas encuestadas de la comunidad nativa de Tsuntsuntsa el $58.3 \%$ que representa a 14 pobladores, manifiestan tener un nivel de participación malo y que $41.7 \%$ que representan a 10 pobladores manifiestan que el nivel de participación es regular (Figura 3). Estos resultados determinan que en la variable estrategias participativas indican que es necesario identificar las estrategias participativas que permitan contribuir a conservar las prácticas sociales de la Comunidad Nativa Tsuntsuntsa, considerando que la comunidad en mención tiene un gran desafío en estas 3 dimensiones para conservar sus prácticas sociales.

Asimismo los resultados de la aplicación del instrumento de la variable prácticas sociales, en relación a la dimensión saberes prácticos se identificó que de las 24 personas encuestadas de la comunidad nativa de Tsuntsuntsa el $66.7 \%$ que representa a 16 pobladores de la comunidad manifiestan tener un nivel malo en la dimensión saberes prácticos sociales y que $33.3 \%$ que representan a 8 pobladores manifiestan que el nivel de participación es regular (Figura 4). Con respecto a la dimensión habilidades culturales se evidenció que de los 24 pobladores encuestados de la comunidad nativa de Tsuntsuntsa el $100 \%$ manifiestan tener un nivel malo en esta dimensión (Figura 5). Y con respecto a los resultados de la dimensión materialidades se evidenció que de los 24 pobladores encuestados de la comunidad nativa de Tsuntsuntsa el $79.2 \%$ señalan tener un nivel bueno de materialidades y el $20.8 \%$ manifiestan tener un nivel excelente (Figura 6).

Según Carmen (2013), las estrategias participativas constituye un conjunto de acciones integradoras e integradas entre sí, basadas en interacción y realizadas por los aprendices con la orientación del instructor. Por lo expuesto las estrategias participativas es un procedimiento cuya aplicación permite seleccionar, evaluar, persistir o abandonar determinadas acciones para llegar a conseguir la meta que nos proponemos. La participación consiste en intervenir de modo activo en el proceso. Por tanto, toda participación implica necesariamente la actividad del que participa. Una estrategia no detalla ni prescribe totalmente el camino que debe seguirse para alcanzar una meta. La estrategia es un procedimiento general que puede ser utilizado en 
muchas y variadas situaciones.García (2004), sostiene que las estrategias participativas tiene mucho que ver con la participación social ya que son aquellas iniciativas sociales en las que las personas toman parte consciente en un espacio, posicionándose y sumándose a ciertos grupos para tener presencia en la esfera pública y así reclamar situaciones o demandar cambios.

De Gortari (2000), manifiesta que las prácticas sociales se refieren a un conjunto de trabajos, acciones, ejercicios, que se realizan constantemente dentro de una sociedad. Unasociedad es definida por sus prácticas sociales, sabiendo que a través de las prácticas sociales el hombre da sentido a los problemas fundamentales de la ciencia, sometiéndolos a las complejas relaciones entre ellos y su entorno. Mediante las prácticas sociales se amplía la experiencia y se penetra en aquellas cualidades de los procesos que no se muestran de un modo aparente. Considerando que las prácticas sociales se ejercen por lo general en situaciones extraescolares y escolares que pueden ser motivadas por contextos políticos, sociales, culturales, ideológicos o de otra naturaleza. Por tanto, una práctica no se limita sólo al conocimiento, aun cuando este último sea el centro de la primera. Por lo que estos resultados indican que la dimensión materiales se encuentra en un porcentaje alto de nivel bueno, sin embargo a ello no se debe dejar de lado esta dimensión, considerando ser necesario identificar las estrategias participativas que contribuyan a conservar las prácticas sociales de la Comunidad Nativa Tsuntsuntsa, haciendo mención que la comunidad nativa de Tsuntsuntsa tiene un gran desafío en estas dimensiones para conservar sus prácticas sociales, considerando que las prácticas sociales son importantes ya que son propias (es decir, las que desarrolla cada persona en el seno de su comunidad) no deben entenderse como las correctas o las adecuadas, ni pensar que las prácticas de otras comunidades están equivocadas. Todo ser humano vive integrado en el contexto de un grupo, forma parte de una sociedad concreta marcada por una cultura, considerando que cada sociedad construye sus propias prácticas en el tiempo y éstas constituyen apenas una manera de hacer las cosas, influenciada por diversos factores.

\section{CONCLUSIONES}

$\mathbf{1}^{\mathbf{0}}$. Del Porcentaje de los niveles de las actividades preparatorias, se evidencia que el $62.5 \%$ perciben un nivel malo y que el $0 \%$ manifiestan que el nivel de la dimensión actividades preparatorias es excelente.

$\mathbf{2}^{\mathbf{0}}$. Del porcentaje de los niveles de exposición de conocimientos, se evidencia que el $66.7 \%$ perciben un nivel malo y que el $0 \%$ el nivel de la dimensión exposición de conocimientos es excelente.

$\mathbf{3}^{\mathbf{0}}$. Del porcentaje de los niveles de participación, se evidencia que el $58.3 \%$ perciben un nivel de participación malo y que el $0 \%$ manifiestan que el nivel de la dimensión participación es excelente.

$4^{\mathbf{0}}$. Del porcentaje de los niveles de los saberes prácticos, se evidencia que el $66.7 \%$ perciben un nivel malo y que el $0 \%$ manifiestan que el nivel de la dimensión saberes prácticos es excelente.

$5^{\circ}$. Del Porcentaje de los niveles de habilidades culturales, se evidencia que el $100 \%$ perciben un nivel malo de la dimensión habilidades culturales.

$\mathbf{6}^{\mathbf{0}}$. Del porcentaje de los niveles de materialidades, se evidencia que el $0 \%$ perciben un nivel malo y el $20.8 \%$ manifiestan que el nivel de la dimensión materialidades es excelente.

$7^{\circ}$. Los pobladores de la comunidad nativa de Tsuntsuntsa, demuestran estrategias participativas y prácticas sociales en diferentes niveles predominando el nivel malo.

\section{REFERENCIAS BIBLIOGRÁFICAS}

Carmen, E. (2013). Implementación de estrategias participativas para mejorar la comprensión lectora en los alumnos(as) del sexto grado " $B$ " de educación primaria de la Institución educativa "Fe y Alegría N49" - Piura 2012 (Tesis de Maestría). Universidad de Piura. Piura, Perú. Recuperado el 28 de noviembre d e $2018, \quad d$ e https://pirhua.udep.edu.pe/bitstream/handl e/11042/1811/MAE_EDUC_103.pdf

De Gortari, E. (2000). Diccionario de la lógica. México, Plaza y Valdés.

García, J. (2004). Políticas y programas de participación social. Madrid: Editorial Síntesis.

Hernández, S., Fernández, C., \& Baptista, L. (2006). Metodología de la Investigación. México: Mc. Graw Hill.

Murillo, J., \& Martínez, C. (2010). Investigación etnográfica. Recu perado de: http://www.uam.es/personal_pdi/stmaria/j murillo/InvestigacionEE/Presentaciones/C urso_10/I_Etnografica_Trabajo.pdf

Sierra, M. (2012). Métodos Generales. Universidad Autónoma del estado de Hidalgo. 